\title{
On the importance of examining the relationship between shape data and biologically meaningful variables. An example studying allometry with geometric morphometrics
}

\author{
Jesús MARUGÁN-LOBÓN*, David BLANCO MIRANDA, Beatriz CHAMERO \& Hugo \\ MARTÍN ABAD
}

Unidad de Paleontología, Dpto. Biología, Universidad Autónoma de Madrid, 28049 Cantoblanco (Madrid), Spain; jesus.marugan@uam.es; david.blanco@estudiante.uam.es; beatriz.chamero@uam.es; hugo.martin@uam.es

* Corresponding author

Marugán-Lobón, J., Blanco Miranda, D., Chamero, B. \& Martín Abad, H. 2013. On the importance of examining the relationship between shape data and biologically meaningful variables. An example studying allometry with geometric morphometrics. [Importancia de investigar la relación entre la forma y variables con sentido biológico. Un ejemplo estudiando la alometría con morfometría geométrica]. Spanish Journal of Palaeontology, 28 (2), 139-148.

Manuscript received 30 April 2013

Manuscript accepted 20 July 2013

(C) Sociedad Española de Paleontología ISSN 2255-0550

\section{ABSTRACT}

Geometric morphometrics (GM) is a tool for the statistical analysis of shape on Cartesian landmark coordinates. However, because GM studies commonly focus on the description of morphological trends within shape space (or morphospace), the predictive power of multivariate statistics to understand morphological change remains underutilized. Here we show the protocols to study allometry in 3D with these tools on a postnatal growth series of the domestic chicken. We contrast three approaches: a 'traditional' one in which size variables are compared statistically, a Principal Components Analysis on size and shape scores (Procrustes form space), and a multivariate regression. In the latter approach we further used three different independent factors inherently related to ontogeny: skull centroid size, body weight, and age of the specimens. The results clearly stress the importance of studying shape change in relation to different causal factors (i.e., with regressions), demonstrating that, indeed, any independent variable or variables that make biological sense can be used to understand morphological change with GM.

Keywords: Gallus gallus, skull shape variation, 3D landmarks, Principal Components Analysis, multivariate regression.

\section{RESUMEN}

La morfometría geométrica es una herramienta para el análisis estadístico de la forma con coordenadas cartesianas claves o landmarks. Sin embargo, como la morfometría geométrica se centra normalmente en la descripción de tendencias morfológicas dentro de un morfoespacio, no se suele aprovechar el poder predictivo de la estadística multivariante para comprender el cambio morfológico. En este trabajo presentamos los protocolos para estudiar mediante estas herramientas la alometría en 3D de una serie de crecimiento postnatal de pollos domésticos. Comparamos tres aproximaciones diferentes: una estadística "tradicional" comparando variables de tamaño, un análisis de componentes principales de valores de tamaño y forma (espacio de forma Procrustes) y una regresión multivariante. Para esta última utilizamos tres factores independientes e inherentemente relacionados con la ontogenia: el tamaño del centroide del cráneo, el peso corporal y la edad de los especímenes. Los resultados destacan la importancia de investigar los cambios de forma en relación con factores causales (p.ej., utilizando regresiones), y demostrar, de hecho, que cualquier variable o variables con sentido biológico pueden ser utilizadas para comprender el cambio morfológico mediante la morfometría geométrica.

Palabras clave: Gallus gallus, variación de forma del cráneo, landmarks 3D, análisis de componentes principales (PCA), regresión multivariante. 


\section{INTRODUCTION}

Professor Nieves López was a polymath, never afraid to ask questions in her incessant thirst for knowledge. Nieves wasn't a user of Geometric Morphometrics (GM) but, while attending a talk on the topic, she expressed her curiosity about how allometry could be so easily analyzed with GM. In particular, she asked, how can it be that size relates to shape data if size has been set aside from the landmark configurations with Procrustes methods? Newcomers who begin to use GM also find this confusing. Indeed, GM allows testing the covariation of shape data not only with size, but with any biologically relevant, independent variable, or variables, with multivariate regression (Monteiro, 1999), as well as more complex models such as the Two-Block Partial Least Squares (Rohlf \& Corti, 2000). It is actually the multivariate analysis of Procrustes data that truly opens a myriad of new possibilities for morphological discovery (Roth \& Mercer, 2000). The fact that shape variation may be predictable by size or any other variable or variables further touches on a key operational point of GM, and is why GM is considered a tool for the statistical analysis of form on Cartesian landmark coordinates (Mitteroecker \& Guntz, 2009). To explain this, here we will use a straightforward example to show how to analyze the relationship between size and shape (allometry) on three-dimensional data of skull growth in the domestic chicken.

The relatively new morphometric tools based on landmark coordinates were labeled geometric because configurations of homologous landmarks -Cartesian coordinates- represent the geometry of forms (Bookstein, 1991), not the forms themselves (i.e., all qualitative information is lost). The application of GM involves the treatment of such landmark data in a two-step procedure: (1) the acquisition of shape data per se, applying Procrustes methods (Gower, 1975; Chapman, 1990); this is the comparative step, and (2) the analysis of the obtained shape data (the Procrustes coordinates) with multivariate statistics, which allows the analysis of the variance among multiple shapes, trends in the data, as well as how such variance may relate to -or is explained by- other biologically meaningful variables. Allometry is a biological example of the latter case, where shape variance is related to size changes.

Different growth rates of parts of an organism result in different organismal forms which is why allometry is so meaningful to the evolution of morphology and shape (Thompson, 1917; Huxley, 1932; Gayon, 2000). Homologous (or biologically comparable) size measurements, such as lengths, weights, or volumes from a sample of organisms, are often linearly correlated (Klingenberg, 1996). This can easily be shown graphically, because allometry is equivalent to a power function (Huxley, 1932); when two measurements are linearly correlated within a Cartesian coordinate system it is logically because both measurements increase in unison (Fig. 1). However, there may be differences in this scaling relationship. When the bivariate relationship is rectilinear both variables scale equivalently and the phenomenon is interpreted as isometric growth (Strauss, 2010), whereas if the scaling between two variables is asymptotical (i.e., exponential) the rate at which one of the measurements changes relative to the other is uneven; by definition this is a case of anisometry (Fig. 1). Allometry allows equating growth and evolution to deviations from a linear relationship (e.g., positive and negative residuals of a line); the latter derive either from changes in the slope or the intercept (Shingleton \& Frankino, 2013). However, it is important to note that a non-rectilinear relationship between two measurements may be due to the fact that the dimensionality between the variables is different. This occurs, for instance, when a linear length is compared to an area or a volume (Gould, 1977), but it may occur in instances when the structure is modular and different units grow with different dynamics (Bastir \& Rosas, 2009). In traditional morphometrics the most common way to deal with this issue is the transformation of the original variables into logarithms, which maintains a straightforward interpretation of the allometric relationship (Huxley, 1932).
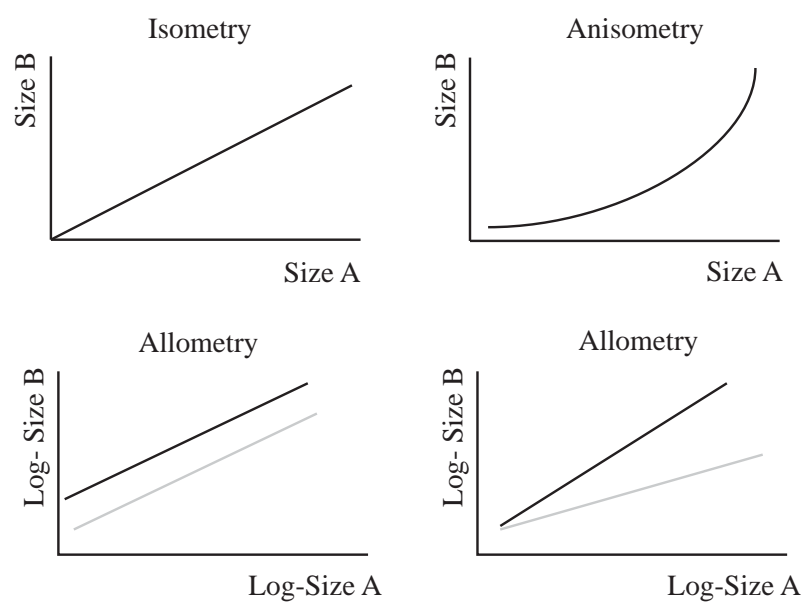

Figure 1. Examples of classical 'Huxelyan' models of isometry, anisometry and allometry with traditional morphometrics. Below are (left) differences in the intercept and (right) on the slope. A, B are morphological traits.

The interpretation of allometry in GM is different from other methodologies in the sense that isometric size has been set aside from the landmark configurations in the shape data (i.e., the configurations are equally scaled; see Methods). Therefore, allometry in GM can only be analyzed in the unique sense of testing if any variation in shape is correlated with size (Strauss, 2010). Thus, 
there is no evaluation of slopes or intercepts, all of which measure the linear association between two variables following specific growth models such as the classic models discussed by Huxley (op. cit). On the other hand, the "real" size of a specimen in GM is represented by the size of each landmark configuration, and this is captured as its 'Centroid Size' (CS), a scalar that accounts for the real distance (in the real scale of each specimen) between the landmarks to the centroid of the configuration (the geodesic center of the configuration). CS is easy to calculate and it is uncorrelated with shape change unless there is allometry, whereas in other cases such as weight or volume, this may or may not be the case (Zelditch et al., 2012). Under these particular circumstances, CS is therefore the most reliable estimate of size in GM, because it provides an unbiased estimate of allometry. However, the fact that CS is so easy to obtain and so mathematically practical does not dismiss other continuous variables inherently related to growth to account for predictable changes in shape (Monteiro, 1999), such as the size of the entire organism (e.g., body mass) and age, as we will show later.

The topic of allometry in biology is too broad for a detailed evaluation here (e.g., Gould, 1966, 1971, 1977; Klingenberg, 1996; Gayon, 2000), and so is the field of geometric morphometrics (Zelditch et al., 2012). For simplicity, we will therefore focus this GM study on two main objectives: (1) showing the protocols to study allometry, and (2) demonstrating how, indeed, GM allows testing the covariation of shape change with any quantitative and independent variable that make sense for a particular biological problem. For the first goal, we will apply the Procrustes form space method (Mitteroecker et al., 2004, 2005) and multivariate regression (Monteiro, 1999). For the second, and given that we will be dealing with a growth series, we will simply test if different scalars related to ontogeny (i.e., skull CS, body weight, and age of the specimens) yield equivalent results, as they should, and discuss the different protocols for the practical study of allometry.

\section{MATERIALS AND METHODS}

\subsection{Sample}

The studied sample for this work consists of a post-natal growth series of $n=37$ skulls of the domestic chicken (Gallus gallus) (Table 1) which was kindly donated by COBB S.A. from natural casualties on their farms (i.e., by stress, not illness). Given that the animals came from different farms, hence different breeds, and places and ages of death were random, we considered skulls as belonging to a random sampling of the same species. Before preparation, each frozen corpse was weighed with a precision balance (Table 1). The skulls were dissected from the corpses, then gently skeletonized manually, and finally dried out. Once prepared, a series of $\mathrm{p}=37$ 3D landmarks (out of which 13 are repeated bilaterally) were digitized on each skull (Table 2; Fig. 2) with a 3D Microscribe digitizer. Digitizing error was controlled by re-digitizing the landmarks on different days (three times) and averaging the coordinates for each specimen. The landmark series were designed to completely cover the entire skull, thus outlining all views of the skull's external anatomy, including the facial skeleton (beak and maxillary portions) and the entire neurocranium.

Table 1. Sex, age (A), centroid size (CS), and weight (W) of the studied specimens.

\begin{tabular}{cccc} 
Individual & A (Week) & CS & W (g) \\
\hline \hline M & 2 & 78.91 & 100 \\
M & 2 & 75.44 & 120 \\
M & 2 & 86.39 & 430 \\
F & 2 & 73.41 & 190 \\
F & 2 & 70.69 & 123 \\
F & 2 & 67.74 & 134 \\
F & 2 & 72.79 & 140 \\
F & 2 & 73.00 & 151 \\
F & 2 & 77.52 & 215 \\
F & 2 & 79.69 & 288 \\
M & 2 & 72.92 & 135 \\
F & 2 & 78.36 & 215 \\
M & 3 & 74.27 & 162 \\
F & 3 & 76.52 & 100 \\
F & 3 & 85.15 & 200 \\
F & 3 & 79.06 & 200 \\
M & 3 & 90.79 & 437 \\
M & 3 & 83.36 & 257 \\
M & 3 & 90.50 & 660 \\
M & 3 & 96.36 & 561 \\
M & 3 & 95.07 & 487 \\
M & 3 & 90.19 & 437 \\
M & 4 & 85.05 & 387 \\
M & 5 & 105.72 & 1150 \\
M & 5 & 96.66 & 502 \\
F & 5 & 99.06 & 525 \\
F & 5 & 101.71 & 825 \\
F & 5 & 94.98 & 672 \\
F & 5 & 97.40 & 750 \\
F & 5 & 97.45 & 670 \\
F & 5 & 103.73 & 850 \\
F & 5 & 99.14 & 670 \\
F & 5 & 113.84 & 1375 \\
F & 5 & 109.64 & 1150 \\
F & 5 & 77.24 & 215 \\
M & 6 & 126.25 & 1400 \\
M & 6 & 128.68 & 1950 \\
\hline F & & \\
\hline
\end{tabular}

M=Male, F=Female 
Table 2. Landmark descriptions.

\begin{tabular}{l|l}
\multicolumn{1}{c}{ Landmark } & \multicolumn{1}{l}{ Description } \\
\hline 1 & Sagittal edge of occipital crest \\
2 & Junction supraoccipital-parietal \\
3 & Suture between parietal-frontal \\
$4^{*}$ & Point of maximal curvature orbit \\
$5^{*}$ & Suture Frontal-nasal-lacrimal \\
$6^{*}$ & Naso-lacrimal suture \\
$7^{*}$ & Nare’s dorsal vertex \\
8 & Medial suture at landmark 7 \\
$9 *$ & Nare's anterior vertex \\
$10^{*}$ & Ventral naso-maxillary union at nare \\
11 & Tip of the beak (premaxilla) \\
$12^{*}$ & Naso-maxillary suture antorbital fenestra \\
$13^{*}$ & Lateral suture at palatine-pterygoid \\
$14 *$ & Orbital process \\
$14^{*}$ & Postorbital process \\
$16^{*}$ & Quadrate articulation w/cranium \\
$17^{*}$ & Jugal articulation at quadrate \\
18 & Medial dorsal margin foramen magnum \\
19 & Exo-occipital-supraoccipital suture \\
20 & Lateral depression at occipital condyle \\
21 & Subcondylar fossa \\
22 & Tuba tympanica \\
$24 *$ & Union at palatine, pterygoid and sphenoid \\
\hline \hline &
\end{tabular}

*Reflected bilaterally

\subsection{Shape Data, step 1}

To obtain the shape data the configurations of landmarks were superimposed using the Generalized Procrustes method, also known as General Procrustes Analysis (GPA; Adams et al., 2004) which is based on a generalized least-squares minimization of the distance between corresponding landmarks (Gower, 1975). Whether in 2D or 3D, the configurations of landmarks are compared by this superimposition, and three maneuvers are required to this end: translation, scaling, and rotation. First, translation implies that all the configurations are brought to a common coordinate system which, by consensus (and because of the mathematical axiomatic properties that it provides) is the average configuration (or grand mean). In a second step, the configurations are rigidly scaled to the same size (i.e., isometric scaling), and the scaling choice is Centroid Size $=1$ (i.e., unit centroid size). Thirdly, the aim is to reduce at maximum the distance between homologous landmarks, and all the configurations are rotated over their shared geodesic centroid. After these three operations the corresponding landmarks will be much closer to each other, but some mismatch will remain. This residual mismatch and irreducible distance among homologous landmarks after the Procrustes alignment is due to the geometric differences between the configurations (after translation, rotation, and scaling have been set aside), and is known as Procrustes shape data. This data is 'invariant' to (i.e., it does not possess any information about) translation, rotation, and scaling, and consists of a data matrix in
Dorsal

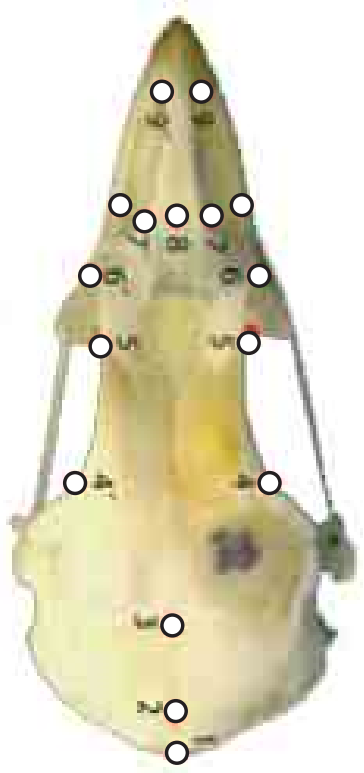

Lateral

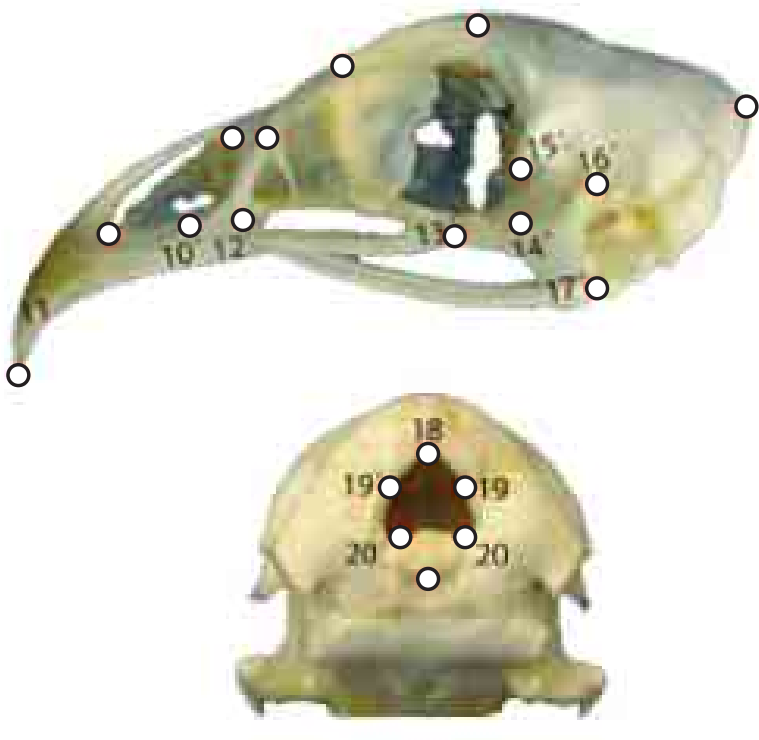

Caudal
Ventral

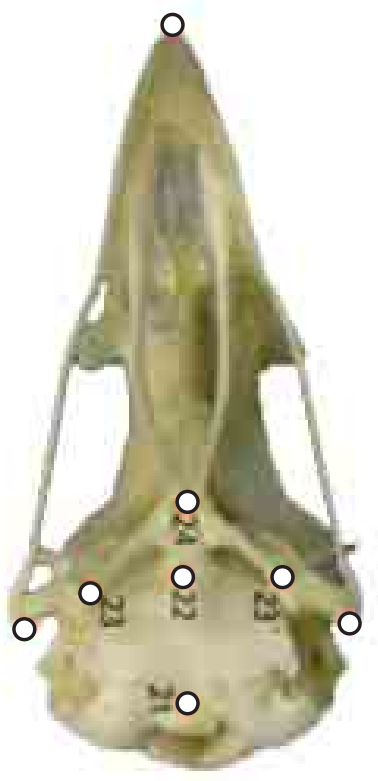

Figure 2. Example of the studied 3D landmark configuration on a chicken skull in four different views. 
which coordinate values for each landmark of each configuration correspond to their location in this newly generated coordinate system (the Procrustes shape space; Dryden \& Mardia, 1998). More mathematical, practical, and historical accounts about the methods can be found in Slice (2007), Mitteroecker \& Guntz (2009), Viscosi \& Cardini (2011), and Zelditch et al. (2012).

In this study, all the GM procedures were performed with the program MorphoJ (v. 1.6.0_27; Klingenberg, 2011) with the exception of Procrustes form space (see below). A special option for the Procrustes treatment of the landmark configurations with MorphoJ is isolating a component of symmetric shape variation which accounts only for the variation of a configuration of landmarks with symmetry (such as a skull) (Mardia et al., 2000). This method, informally called 'symmetrization', yields a component of shape variation among individuals in what might be considered a left-right averaging (Savriama et al., 2012). This helps ignoring any source of variation within the sample due to asymmetry (which is necessary only for biological studies interested in analyzing that factor), and implicitly entails reducing the small yet potentially present error introduced by the separate digitizing of two nearly identical sides of a symmetrical form.

\subsection{Statistical Analyses, step 2}

There are two ways to test for allometry using GM. One approach proposes to add a column of natural logtransformed CS variables within a principal components analysis (PCA) accompanying the Procrustes data matrix (Mitteroecker et al., 2004, 2005). The method was originally called Size-Shape-space, but its similitude with Dryden \& Mardia's (1998) Size-and-Shape space -the space that results by superimposing landmark configurations without re-scaling them- led to coin the name Procrustes form space at the Vienna Morphofest in July 2006, and to our records, the first citation applying it was by Bastir et al. (2007). Analytically, PCA summarizes large amounts of data in a new set of variables (eigenvectors), which are a linear combination of the original variables in decreasing order of amount of explained variance (Lattin et al., 2002). By incorporating size in the shape data-matrix, in case of a meaningful association between size and shape, such size-shape PCA will yield an ordination (Procrustes form space) in which the first dimension captures the covariation between size and shape (allometry), subsequently yielding orthogonal dimensions (PCs) of less-explained shape variance, which will therefore be completely independent of size. Besides the platform R (user-programmable), so far there are only two programs available to perform a form space (or sizeshape) analysis, TPSrelw (for 2D data) and Morphologika (for 3D). However, Procrustes form space can be computed in any statistical package, such as PAST(C), Statistica $(\subset$ or SPSS $\odot$, simply exporting the Procrustes shape data and centroid size, say, from MorphoJ (using the Ln-transform of CS, and adding this to the last column of the shape datamatrix before performing a variance-covariance PCA). Thereafter, shape data can be regressed on the obtained PC-scores to visualize the meaning of such scores as shape changes. Here, we performed the PCA with Statistica $₫$ (v. 8.0; Statsoft Inc., 2007).

Multivariate regression can also be used to test the association between size and shape data (Monterio, 1999). In our case study the independent (predictor variable) can be CS or log-CS, but it can also be mass (W) or age (A) (Table 1). For the study of allometry, one variable to test with the regression model would be CS, as this scalar represents the real size of the skull for each specimen. As in other software, MorphoJ predicts a vector which is equivalent to a shape variable that is most strongly associated with the independent variable (Drake \& Klingenberg, 2008), and the statistical significance of its explained variance (a multivariate equivalent to $R^{2}$ ) is measured by permutation (here, 10,000 rounds). In order to graphically show the relationship between the independent variables and shape, MorphoJ provides a scatter-plot of the regression scores (ordinate) on the independent variable (abscissa) for all the observations in the sample. This set of regression scores, just as the scores of the principal component's ordination, are obtained by projecting each individual's data onto the obtained regression vector. If the resulting association is curved, it can be useful to linearize the data using log-CS (i.e., the natural logarithm). This is important because the implemented statistical models test for a rectilinear correlation between shape and independent data. Thus, a correct interpretation of the significance of the predicted shape changes will only make sense if there is a rectilinear relationship between size and shape. For the same reason, $\mathrm{W}$ (weight) and A (age) may or may not need to be log-transformed, but this may only be determined after a preliminary analysis of the data.

On the other hand, the use of CS as a predictor variable addresses how skull shape changes relative to skull size. However, using body mass as an independent variable addresses how skull shape changes relative to changes in the size of the entire animal. This difference is important because skull size may scale differently to the rest of the body, and this surely affects skull shape as a whole (Gould, 1966). In our example this could be the case, for instance, between sexes, since males or females could have relatively smaller or larger skulls compared to the other sex. Therefore, some skull-shape differences may be related to this sexually dimorphic allometric shift. When A (age) is used as an independent variable, the question addressed is if skull shape changes through post-natal time, which is obviously assumed to be the case (the null hypothesis). Interestingly, Ponce de León \& Zollikofer (2001) argued 
that these situations may be typified differently; using size and shape in combination would entail studying ontogenetic allometry, while combining shape data and time would be related to studying development (though in our case, this would be post-natal).

Visualization is one of the greatest advantages of using GM, being able to show shape differences on virtual models that closely resemble the studied forms (Rohlf, 1993). Differences in shape (or shape differences correlated to size) correspond to differences in the coordinates of landmarks, and in GM these are visually expressed as deviations of the landmarks relative to the mean configuration. For comparison, it is therefore necessary to have two configurations, a reference and a target. These can either be two specimens of the sample; or the mean configuration (the consensus configuration) as the reference, and a shape estimated statistically, for example after performing a PCA or a regression, as the target. Shape differences are usually shown either with vectors denoting the displacement of the landmarks from the reference to the target, or by deforming an orthogonal grid over the reference to the target configuration, simulating D'Arcy Thompson deformation grids (Thompson, 1917). The latter deformation is attained by a smoothing equation known as the Thin Plate Spline (TPS; Bookstein, 1989), which computes the estimated location of coordinates surrounding the landmarks necessary to deform the grid from the reference to the target. Following the same logic and using the TPS, it is possible to deform images by estimating the pixels surrounding the landmarks from one configuration onto another (image unwarping; Bookstein, 1989; Rohlf, 2002) and, with less graphical distortion, estimating the corresponding polygons of 3D meshes rendered by CTscans, laser-scans, and photogrammetry. Deforming a virtual, yet real-looking form, out of a polygonal mesh was computer-exhausting less than a decade ago, but with today's computers this is becoming a standard practice in GM, clearly providing the most graphically appealing and easy to interpret results. Here we used the software Landmark v. 3.6 (http://www.idav.ucdavis.edu/research/ EvoMorph) to perform these depictions.

\section{RESULTS}

The analysis of the independent variables CS, W, and A is equivalent to a 'traditional' morphometric analysis (see Adams et al., 2004) to evaluate allometry, because CS is a size scalar, as it can be W. However, CS is rarely compared to other variables in this way. Interestingly, the correlation between CS and W is slightly curved (even though CS here is three dimensional, hence both being dimensionally congruent; Fig. 3a), entailing that skull size (denoted by CS) grows at faster rates in earlier stages (first week) relative to the achievement of larger body masses (i.e., allometrically) than later. This notwithstanding, body mass (W) was log-transformed because it was right skewed (histogram; Fig. 3a), and CS was therefore also log-transformed to maintain all the data dimensionality congruent. All the independent variables, skull Log-CS and body mass (Log-W) and age (A) are positively correlated (Fig. 3b), logically entailing that both the size of the skull and body mass increase as time passes.

a)

Original Variables
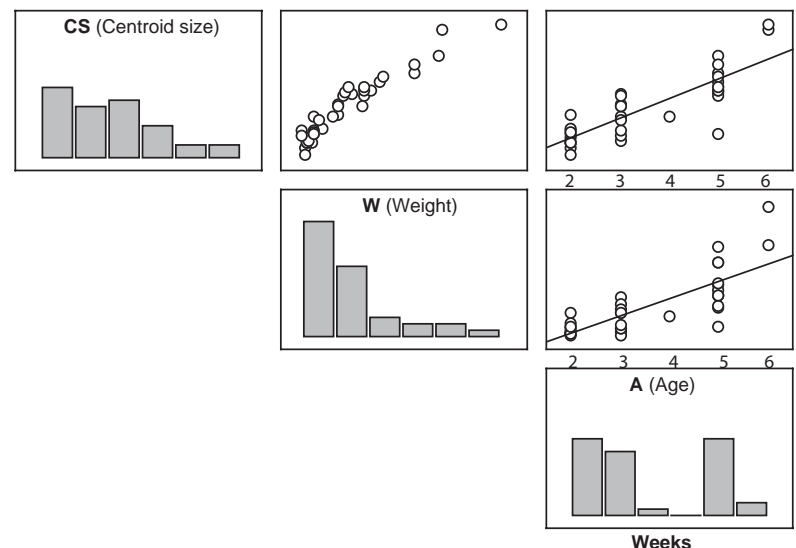

b)

\section{Log-transformed Variables}
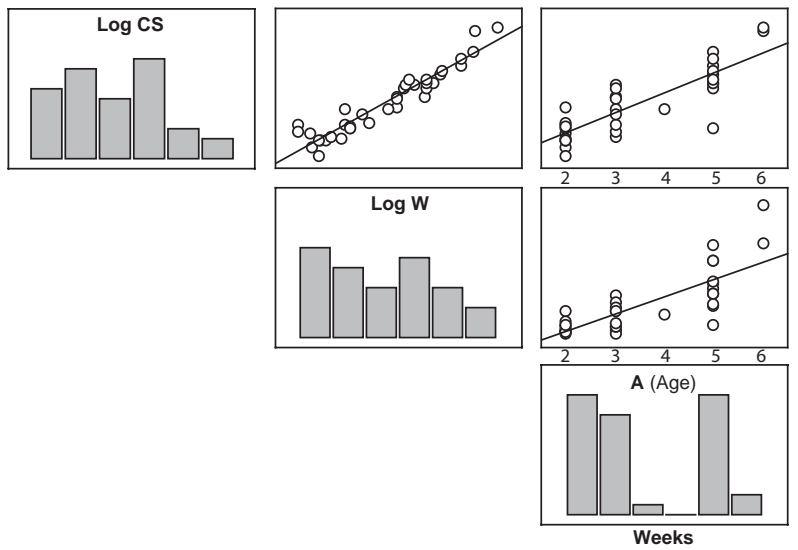

Figure 3. Correlation matrices of independent variables (CS, $\mathrm{W}$, and A, and Log-transformed). a) Original values, b) log-transformed values. Histograms show how the frequency distributions change before and after logtransformation.

The multivariate analyses indicate that post-natal growth in the chicken skull is clearly related to shape changes of the entire facial skeleton (its relative growth) and to minor changes in the cranium, which are related with a depression of the cranial roof. The first eigenvector 
of the PCA including size-shape data (PC1 of Procrustes form space), explained more than $92 \%$ of the variance. Notice, however, that out of this PCA it is impossible to know how much shape variance is explained by the first dimension, because the results includes size, which is the variable that clearly increases the explained variance to more than $90 \%$. In fact, the only way to know how much shape variance per se corresponds to this dimension is by regressing shape to the obtained PC1 scores using a multivariate linear regression. We performed this analysis and found that PC1 explains $33 \%$ of the total variance (p>0.001; Fig. 4).

Using multivariate regressions, the independent variables Log-CS, Log-W, and A explain the same shape changes as the latter PC (compare Figs 4-5), predicting $33 \%, 28.90 \%$ and $23.03 \%$ of the explained variance, respectively (all, $\mathrm{p}<0.001$ ). These percentages are relatively equal, though the exception is age (A). The farmers provided age data in weeks, which is a relatively imprecise measure of time to study ontogenetic change (i.e., individual growth rates can vary a lot within a week on a daily basis). This time bias explains shape variance by $\mathrm{A}$ is lower than the rest (ca. $10 \%$ less, which is a small difference anyway), and why the grey-scale color coding of the points within the 3D scatter (Fig. 5) shows a great deal of overlapping.
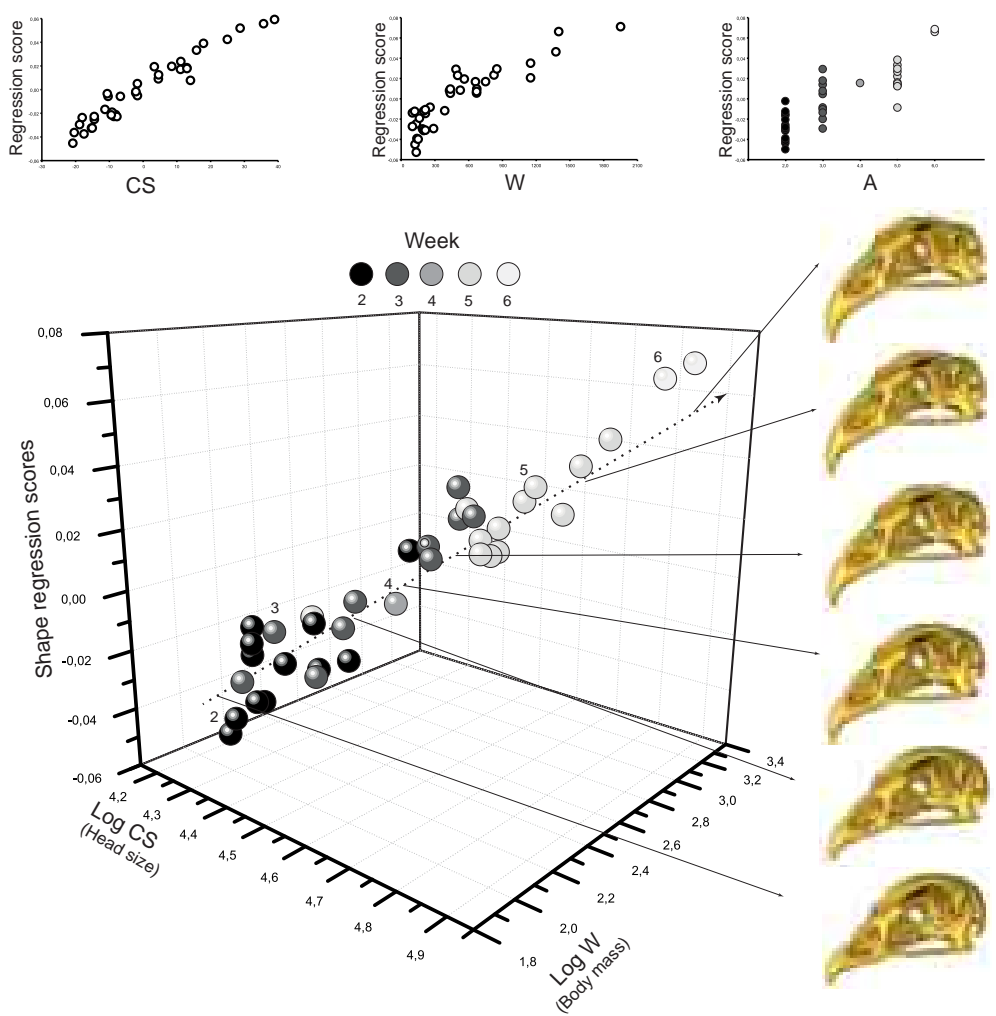
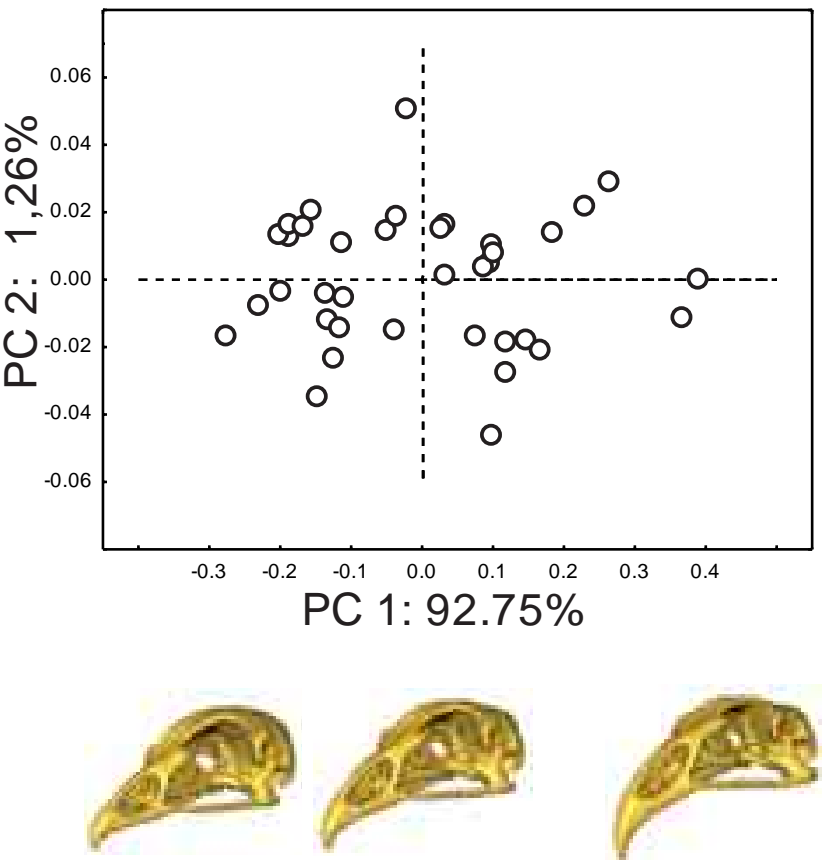

Figure 4. Procrustes form space scatter-plot (PCA of shape and size data). Notice that the axes of the scatterplot are equally scaled to each other for visualization purposes (i.e., PC1 should be much longer than PC2). The skulls show the skull shape differences accounted by PC1 scores as a warped (averaged) CT-scan, and were obtained by egressing the shape data on the PC1 scores. Notice that these shape changes are identical to those predicted by any of the rest of variables with the regression models.
Figure 5. Skull-shape variance prediction by CS, W, and $\mathrm{T}$ (above), and correlation between shape regression vector ( $\mathrm{x}$ and $\mathrm{y}$ ) with shape on the z-axis. Skull differences shown as a warped (averaged) CT-scan of a chicken skull corresponding to the predicted scores by the multivariate regression (here, those estimated by Log-CS, but Log_W and A are identical; see text). 


\section{DISCUSSION}

More than a decade ago Monteiro (1999) discussed the usefulness of combining multivariate regression with GM for morphological research. At that time the combination of these methods and GM were still underutilized perhaps because they seemed either counterintuitive but, surely, complicated. Today the use of GM is very much extended in morphological research, yet most studies still focus on morphospace construction and comparative investigations, and only few exploit GM and its predictive multivariate toolkit to a full potential. In most cases, the causes for this limited use of GM together with complex statistical modeling may still be the same today as it was a decade ago. Here we have shown how useful and straightforward it is to exploit together GM and multivariate regression (as well as any other predictive multivariate technique, such as the Two-Block Partial Least Squares; Rohlf \& Corti, 2000) for biological enquiry on morphological change (Marugán-Lobón, 2010).

Using a simple example, a chicken growth series, we asked if a set of three variables inherent to growth, the size of the skull as the centroid size of its landmark configuration (CS), body mass (W) and age (A), could predict skull shape changes in the chicken's skull, and what these shape changes were. Intuitively, the three variables should predict the same pattern and unsurprisingly this is what the results have shown; as chickens grow most shape changes take place in the facial skeleton, with slight changes in the cranial roof. Interestingly, only ca. $30 \%$ of skull shape change in chicken growth is explained by size or age, entailing that the rest of shape differences among skulls are non-allometric. Given that we are studying a growth series, these shape differences between individuals presumably must have emerged earlier in ontogeny. It would be worth asking (again using multivariate techniques and alternative covariates) if the remaining shape variance relates to other aspects of development or the biology of the chicken. For instance, earlier onset of sexual shape dimorphic differences could explain part of the remaining differences in the data. Indeed, we found that there are differences in relative beak size between sexes, though unfortunately, we found that females in the farm were treated such that their beaks were cut at the tip because under stress conditions they tend to attack each other. Landmark analysis is extremely precise, and thus, this could be the reason why we found such shape dimorphism in the relative size of the beak, preventing us from further analyzing -and showing- these results.

Using Procrustes form space or multivariate regression to study allometry depends on the choice of the researcher, although multivariate regression allows a more ample repertoire of biologically meaningful questions to be addressed. Procrustes form space fits well with the conceptual definition of form (form=size+shape; Needham,
1950), and operationally it is practical because it allows comparing developmental trajectories (or growth trajectories) over morphospace (Mitteroecker et al., 2004). However, Procrustes form space does not allow knowing the percentage of shape variance separated from size. Operationally, the CS and Procrustes coordinates (the original shape data) are in completely different scales, which would require using the correlation matrix for the PCA (to standardize the differences between data) instead of the variance/covariance matrix, but would likely yield different and unpredictable projections of the data (Flury, 1997). On the other hand, size in this approach can only be the Ln-CS, and no other variable can be added to the PCA (at least in principle), because only CS fits the assumptions of shape distribution within shape space (Mitteroecker et al., 2004; Mitteroecker \& Guntz, 2008). Thus, as the Size-and-Shape space previously proposed by Dryden \& Mardia (1998), the Procrustes form space is a useful tool to study allometry, although it is limited to testing allometry relative to the particular size of a given set of landmark configurations.

In this study we have shown that there is much to learn from morphological change with geometric morphometrics in combination with multivariate statistics, particularly predictive models. GM is a relatively new technique, and reciprocally, there is also much to learn about GM by the exploitation of the latter tools. For instance, in any study of organismal allometry with GM we still need to understand what that non-allometric component is and how to approach it (Strauss, 2010). Likewise, and importantly, one does not need to stick to the use of CS alone as a causal factor to address morphological change (Monteiro, 1999). In fact -and answering Nieves' question- any study that aims at understanding organismal shape change and morphological evolution can be approached in the same way as we have approached allometry, with multivariate regression and one or multiple independent covariates. The requisite is to take into account that a biologically reasonable hypothesis must link such independent variable or variables to phenotypic change.

\section{ACKNOWLEDGEMENTS}

This paper is part of the papers honoring Nieves, and we thank the editors, for their patience and effort in putting everything together. We would like to thank Juan Carlos at COBB S.A. for providing us with the specimens, Álvaro López for helping in the preparation of the material, and the staff at the San Francisco de Asís Hospital's digital imagery lab for the CT-scans of the chicken skull. The manuscript was greatly improved by comments from Diego Rasskin and Markus Bastir, and is supported by project MCINN CCGL2009-11838BTE. A Nieves López y Santi Reig, aquello que nos faltó deciros. 


\section{REFERENCES}

Adams, D.C., Slice, D.E. \& Rohlf, F.J. 2004. Geometric morphometrics: ten years of progress following the “revolution”. Italian Journal of Zoology, 71, 5-16.

Bastir, M., O’Higgins, P. \& Rosas, A. 2007. Facial ontogeny in Neanderthals and modern humans. Proceedings of the Royal Society B: Biological Sciences, 274, 1125-1132.

Bastir, M. \& Rosas, A. 2009. Mosaic evolution of the basicranium in Homo and its relation to modular development. Evolutionary Biology, 36, 57-70.

Bookstein, F.L. 1989. Principal warps: thin plate splines and the decomposition of deformations. IEEE Transactions on Pattern Analysis and Machine Intelligence, 11, 567-585.

Bookstein, F.L. 1991. Morphometric tools for landmark data: geometry and biology. Cambridge University Press, New York.

Chapman, R.E. 1990. Conventional Procrustes approaches. In: Conventional procrustes approaches (eds. Rohlf, F.J. \& Bookstein, F.). University of Michigan Museum of Zoology, Ann Arbor, 251-267.

Drake, A.G. \& Klingenberg, C.P. 2008. The pace of morphological change: Historical transformation of skull shape in St. Bernard dogs. Proceedings of the Royal Society London B Biological Sciences, 275, 71-76.

Dryden, I.L. \& Mardia, K.V. 1998. Statistical shape analysis. Wiley, New York.

Flury, B. 1997. A First Course in Multivariate Statistics. Springer-Verlag, New York.

Gayon, J. 2000. History of the concept of allometry. American Zoologist, 40, 748-758.

Gould, S.J. 1966. Allometry and size in ontogeny and phylogeny. Biological Reviews of the Cambridge Philosophical Society, 41, 587-640.

Gould, S.J. 1971. Geometric similarity in allometric growth: a contribution to the problem of scaling in the evolution of size. The American Naturalist, 105, 113-136.

Gould, S.J. 1977. Ever since Darwin. W.W. Norton, New York.

Gower, J.C. 1975. Generalized Procrustes analysis. Psychometrika, 40, 33-51.

Huxley, J.S. 1932. Problems of relative growth. Methuen and Co., Ltd., London.

Klingenberg, C.P. 1996. Multivariate allometry. In: Advances in morphometrics (ed. Marcus, L.F.). Plenum Press, New York, 23-49.

Klingenberg, C.P. 2011. MorphoJ: an integrated software package for geometric morphometrics. Molecular Ecology Resources, 11, 353-357.

Lattin, J., Carroll, D. \& Green, P. 2002. Analyzing multivariate data. Duxbury Applied Sciences, Duxbury Press, Pacific Grove, California.

Mardia, K.V., Bookstein, F.L. \& Moreton, I.J. 2000. Statistical assessment of bilateral symmetry of shapes. Biometrika, 87, 285-300.

Marugán-Lobón, J. 2010. Combining Shape Data and Traditional Measurements with the 2B-PLS: Testing the Covariation Between Avian Brain Size and Cranial
Shape Variation as an Example. In: Morphometrics for non-morphometricians (ed. Eleewa, A.). Springer-Verlag, Berlin, 179-190.

Mitteroecker, P. \& Guntz, P. 2009. Advances in morphometrics. Evolutionary Biology, 36, 235-247.

Mitteroecker, P., Guntz, P., Bernhard, M., Schaefer, K. \& Bookstein, F. 2004. Comparison of cranial ontogenetic trajectories among great apes and humans. Journal of Human Evolution, 46(6), 679-697.

Mitteroecker, P., Gunz, P. \& Bookstein, F. 2005. Heterochrony and geometric morphometrics: a comparison of cranial growth in Pan paniscus versus Pan troglodytes. Evolution and Development, 7(3), 244-258.

Monteiro, L.R. 1999. Multivariate regression models and geometric morphometrics: The search for causal factors in the analysis of shape. Systematic Biology, 48(1), 192199.

Needham, J. 1950. Biochemistry and Morphogenesis. Cambridge University Press, Cambridge.

Ponce de León, M. \& Zollikofer, C. 2001. Neandertal cranial ontogeny and its implications for late hominid diversity. Nature, 412, 534-538.

Rohlf, F.J. 1993. Relative warps analysis and an example of its application to mosquito wings. In: Contributions to Morphometrics (eds. Marcus, L.F., Bello, E. \& GarcíaValdecasas, A.). Monografías del Museo Nacional de Ciencias Naturales, CSIC, Madrid, 131-158.

Rohlf, F.J. 2002. Geometric morphometrics in phylogeny. In: Morphology, shape and phylogenetics (eds. Forey, P. \& Macleod, N.). Francis \& Taylor, London, 175-193.

Rohlf, F.J. \& Corti, M. 2000. The use of two-block partial least-squares to study covariation in shape. Systematic Biology, 49, 740-753.

Roth, V.L. \& Mercer, J.M. 2000. Morphometrics in development and evolution. American Zoologist, 40, 801-810.

Savriama, Y., Gómez, J.M., Perfectti, F. \& Klingenberg, C.P. 2012. Geometric morphometrics of corolla shape: dissecting components of symmetric and asymmetric variation in Erysimum mediohispanicum (Brassicaceae). New Phytologist, 196, 945-954.

Shingleton, A.W. \& Frankino, W.A. 2013. New perspectives on the evolution of exaggerated traits. Bioessays, doi: 10.1002/bies.201200139.

Slice, D.E. 2007. Geometric morphometrics. Annual Review of Anthropology, 36, 261-81.

Strauss, R.E. 2010. Prospectus: The Future of Morphometrics. In: Morphometrics for non-morphometricians (ed. Eleewa, A.). Springer-Verlag, Berlin, 345-352.

Thompson, D’A.W. 1917. On Growth and Form. Cambridge University Press, Cambridge.

Viscosi, V. \& Cardini, A. 2011. Leaf Morphology, Taxonomy and Geometric Morphometrics: A Simplified Protocol for Beginners. PlosOne, 6(10), e25630, doi: 10.1371/journal. pone. 0025630.

Zelditch, M.L., Swiderski, D., Sheets, D. \& Fink, W. 2012. Geometric Morphometric for Biologists: A Primer. Elsevier, $2^{\text {nd }}$ Ed., London. 
